\title{
On periodic $L^{2} n$-widths
}

\author{
Michael S. Floater ${ }^{\mathrm{a}}$, Espen Sande ${ }^{\mathrm{a}}$ \\ ${ }^{a}$ Department of Mathematics, University of Oslo, Moltke Moes vei 35, 0851 Oslo, Norway
}

\begin{abstract}
In this paper we study the $n$-width problem for the Sobolev space of periodic functions, $H_{\mathrm{per}}^{r}(0,2 \pi)$. Building on a theorem of Pinkus we show that it admits optimal even-dimensional spline spaces of all degrees $\geq r-1$. Then, by using a theorem of Karlovitz, we show that it does not admit any optimal spline space of odd dimension $>1$.
\end{abstract}

Keywords: $n$-Widths, Splines, Isogeometric analysis, Green's functions 2000 MSC: 41A15, 47G10, 41A44

\section{Introduction}

In computer-aided design, geometric objects are often modelled using parametric spline curves and surfaces, and analysis of the model typically involves solving a PDE. One of the basic ideas of isogeometric analysis (IGA) is to solve these PDEs in a finite element framework where, as far as possible, the elements are chosen to be the spline basis functions used to represent the geometry $[6,2,3,1]$. If necessary, these spline bases can then be refined, by inserting knots, or by raising their degree, in order to generate more degrees of freedom, and achieve a better approximation to the solution of the PDE.

Thus, isogeometric analysis motivates a mathematical study of the approximation power of spline spaces, especially with respect to the $L^{2}$ norm. To do this, we need to restrict our attention to some suitable class of functions to approximate. From the point of view of elliptic PDEs, a natural class of functions to approximate on an interval $(a, b)$ is the Sobolev space

$$
H^{r}(a, b)=\left\{u \in L^{2}(a, b): u^{(\alpha)} \in L^{2}(a, b), \quad \alpha=1,2, \ldots, r\right\} .
$$

Kolmogorov [8] showed that for any $n \geq r$ there is an $n$-dimensional space that is optimal for the $L^{2}$ approximation of functions in this class. The space is spanned by the polynomials of degree at most $r-1$ and the first $n-r$ eigenfunctions of an associated eigenvalue problem. Later, it was shown by Melkman and Micchelli [10] that there are two spaces of splines that are also optimal for this problem, one of degree $r-1$, the other of degree $2 r-1$. Later, we showed in [4] that these two spline spaces are only the first of a sequence of optimal spline spaces of degrees $l r-1, l=1,2,3, \ldots$. Thus, from the point of view of approximating functions in $H^{r}(a, b)$, one can argue that splines of any degree of the form $l r-1, l=1,2,3, \ldots$, with the right knot vector, are equally good. In the important case $r=1$ there are optimal spline spaces of every degree, and the knot vectors are uniform.

For $r \geq 2$ it is an open question whether there are optimal spline spaces of degrees other than of the form $l r-1$. This was a motivation for our paper [5] in which we studied the related problem of approximating functions in $H^{r}(a, b)$ that are restricted to satisfy certain boundary conditions, specifically the condition that derivatives of either odd or even order are zero at the endpoints $a$ and $b$. We were able to show that for these restricted classes of functions, there are indeed optimal spline spaces of all degrees $d \geq r-1$, and, moreover, all their knot vectors are uniform, making them easy to use in practice.

Email addresses: michaelf@math.uio.no (Michael S. Floater), espsand@math.uio.no (Espen Sande) 
In this paper we study an alternative restriction of the Sobolev space $H^{r}(a, b)$, namely periodic boundary conditions. This space is of interest when solving PDEs with periodic boundary conditions. For simplicity, we will fix $(a, b)=(0,2 \pi)$, and we will denote by $H_{\text {per }}^{r}$ the subspace of $H^{r}(0,2 \pi)$,

$$
H_{\mathrm{per}}^{r}=H_{\mathrm{per}}^{r}(0,2 \pi)=\left\{u \in H^{r}(0,2 \pi): \quad u^{(\alpha)}(0)=u^{(\alpha)}(2 \pi), \quad \alpha=0,1, \ldots, r-1\right\} .
$$

Finding optimal spaces for periodic function spaces is a classical problem and various results have been derived. In [12, Chapter IV, Section 6] Pinkus studied the $L^{2}$ approximation of functions $u \in H_{\text {per }}^{r}$, and measured the error relative to the $L^{2}$ norm of $u^{(r)}$ (also called the $H^{r}$ semi-norm of $u$ ). He proved that the $2 m$-dimensional space consisting of uniform, periodic splines of degree $r-1$ is in this case optimal, i.e. minimizes the relative error. In fact, he proved a more general result regarding spaces defined by cyclic variation diminishing operators. In [11] he also showed optimality of this spline space in various other cases of $L^{p}$ approximations to periodic functions with bounded $r$ th derivative in $L^{q}$. See also [12, Chapter V, Section 4].

Evans et al. [3] also studied the problem of $L^{2}$ approximation of functions $u \in H_{\mathrm{per}}^{r}$, but they measured the error relative to the full $H^{r}$ norm of $u$. Based on their numerical results they conjectured [3, Conjecture 5.2] that in this case there is a sequence of optimal $2 m$-dimensional spline spaces, one for every degree $\geq r-1$. Similar optimality results for this sequence have already been shown in other periodic $L^{p}$ settings; see Theorems 8.1.11 and 8.1.13 of [9] for more details.

By applying the aforementioned $L^{2}$ result of Pinkus, together with the techniques developed in [5], we will in this paper partially confirm [3, Conjecture 5.2]. The only difference is that, similar to Pinkus, we study the $L^{2}$ error relative to the $H^{r}$ semi-norm, and not the full $H^{r}$ norm.

\section{Kolmogorov $n$-widths}

We denote the norm and inner product on $L^{2}=L^{2}(0,2 \pi)$ by

$$
\|f\|^{2}=(f, f), \quad(f, g)=\int_{0}^{2 \pi} f(t) g(t) d t,
$$

for real-valued functions $f$ and $g$. Let $A$ be a subset of $L^{2}$, and $X_{n}$ an $n$-dimensional subspace of $L^{2}$. Then

$$
E\left(A, X_{n}\right)=\sup _{u \in A} \inf _{v \in X_{n}}\|u-v\|
$$

is the distance to $A$ from $X_{n}$ relative to the $L^{2}$ norm. The Kolmogorov $L^{2} n$-width of $A$ is then defined by

$$
d_{n}(A)=\inf _{X_{n}} E\left(A, X_{n}\right)
$$

A subspace $X_{n}$ is called an optimal subspace for $A$ provided that

$$
d_{n}(A)=E\left(A, X_{n}\right)
$$

For $r \geq 1$ we define the set of functions

$$
A_{\mathrm{per}}^{r}=\left\{u \in H_{\mathrm{per}}^{r}:\left\|u^{(r)}\right\| \leq 1\right\}
$$

then, if $X_{n}$ is optimal for $A_{\text {per }}^{r}$, we have

$$
\left\|u-P_{n} u\right\| \leq d_{n}\left(A_{\mathrm{per}}^{r}\right)\left\|u^{(r)}\right\|,
$$

where $P_{n}$ is the $L^{2}$ projection onto $X_{n}$ and $d_{n}\left(A_{\text {per }}^{r}\right)$ is the least possible constant over all $n$-dimensional subspaces of $L^{2}$. 


\section{Kernels and convolutions}

Let $K$ be any integral operator

$$
K f(x)=\int_{0}^{2 \pi} K(x, y) f(y) d y,
$$

whose kernel is $K(x, y)$. We denote its adjoint by $K^{*}$, defined as

$$
\left(f, K^{*} g\right)=(K f, g) .
$$

The kernel of $K^{*}$ is given by $K^{*}(x, y)=K(y, x)$.

For $r \geq 1$, consider the function classes $A^{r}$, defined by

$$
A^{r}= \begin{cases}\left(K K^{*}\right)^{i} K(B), & r=2 i+1, \\ \left(K K^{*}\right)^{i}(B) & r=2 i,\end{cases}
$$

where $B=\{f:\|f\| \leq 1\}$ is the unit ball in $L^{2}$. As shown in [5] the $n$-widths of these function classes satisfy

$$
d_{n}\left(A^{r}\right)=d_{n}(A)^{r} .
$$

Furthermore, it follows from [5, Theorem 4] that if $X_{n}^{0}$ is optimal for $A^{1}=K(B)$ and $Y_{n}^{0}$ is optimal for $K^{*}(B)$ then, for each $r \geq 1$, the subspaces

$$
X_{n}^{d}= \begin{cases}\left(K K^{*}\right)^{i}\left(X_{n}^{0}\right), & d=2 i \\ \left(K K^{*}\right)^{i} K\left(Y_{n}^{0}\right), & d=2 i+1,\end{cases}
$$

are optimal for the function classes $A^{r}$ for all $d \geq r-1$. We note that these function classes, and subspaces, were defined in [5] with respect to $L^{2}(0,1)$ rather than $L^{2}(0,2 \pi)$, but [5, Theorem 4] is independent of the length of the interval.

In this paper we will only consider an integral operator $K$ satisfying $K^{*}=-K$. For such a $K$ the function classes $A^{r}, r \geq 1$, have the much simpler representation

$$
A^{r}=K^{r}(B)
$$

since $f \in B$ if and only if $-f \in B$. Moreover, one can obtain the following corollary of [5, Theorem 4].

Corollary 1. Assume $K$ satisfies $K^{*}= \pm K$ and let $X_{n}^{0}$ be optimal for $A^{1}$. Then, for each $r \geq 1$, the spaces

$$
X_{n}^{d}=K^{d}\left(X_{n}^{0}\right)
$$

are optimal for the function classes $A^{r}$ for all $d \geq r-1$.

Proof. $X_{n}^{0}$ plays the role of both $X_{n}^{0}$ and $Y_{n}^{0}$ in [5, Theorem 4].

Let $*$ denote the convolution operator on $[0,2 \pi)$ defined by

$$
(g * f)(x)=\int_{0}^{2 \pi} g(x-y) f(y) \mathrm{d} y,
$$

and observe that if the kernel of $K$ is given by $K(x, y)=g(x-y)$, then $K f(x)=(g * f)(x)$ for $g$ in (1), and so [5, Theorem 4] and Corollary 1 also applies to function classes defined in terms of a convolution. 


\section{Spline spaces}

Suppose $\boldsymbol{\tau}=\left(\tau_{1}, \ldots, \tau_{k}\right)$ is a knot vector such that

$$
0<\tau_{1}<\cdots<\tau_{k}<2 \pi,
$$

and let $I_{0}=\left[0, \tau_{1}\right), I_{j}=\left[\tau_{j}, \tau_{j+1}\right), j=1, \ldots, k-1$, and $I_{k}=\left[\tau_{k}, 2 \pi\right]$. For any $d \geq 0$, let $\Pi_{d}$ be the space of polynomials of degree at most $d$. We then define the spline space $S_{d, \tau}$ by

$$
S_{d, \tau}=\left\{s \in C^{d-1}[0,2 \pi]:\left.s\right|_{I_{j}} \in \Pi_{d}, j=0,1, \ldots, k\right\},
$$

which has dimension $k+d+1$. We will study two kinds of periodic spline spaces. The first is the $(k+1)-$ dimensional space

$$
S_{d, \boldsymbol{\tau}, \mathrm{per}}=\left\{s \in S_{d, \boldsymbol{\tau}}: s^{(\alpha)}(0)=s^{(\alpha)}(2 \pi), \alpha=0, \ldots, d-1\right\} .
$$

The other is the $k$-dimensional space

$$
\hat{S}_{d, \boldsymbol{\tau}, \mathrm{per}}=\left\{s \in S_{d, \boldsymbol{\tau}, \mathrm{per}}: s^{(d)}(0)=s^{(d)}(2 \pi)\right\} .
$$

Observe that the extra boundary condition imposed on $\hat{S}_{d, \boldsymbol{\tau}, \text { per }}$ ensures that $\left.s\right|_{I_{k}}$ is fully determined by $\left.s\right|_{I_{0}}$ (and vice versa) for $s \in \hat{S}_{d, \boldsymbol{\tau}, \text { per }}$.

\section{Optimality of splines of even dimension}

Let us start by considering the uniform knot vector $\boldsymbol{\tau}=\left(\tau_{1}, \ldots, \tau_{2 m-1}\right), m \geq 1$, given by

$$
\tau_{j}=\frac{j \pi}{m}, \quad j=1, \ldots, 2 m-1,
$$

whose associated spline space $S_{d, \boldsymbol{\tau}, \text { per }}$ has even dimension $2 \mathrm{~m}$. We will show that this space is optimal for $A_{\mathrm{per}}^{r}$ for $d \geq r-1$.

We first represent the set $A_{\mathrm{per}}^{r}$ in terms of a convolution. Let the function $D$ be the linear Bernoulli polynomial with respect to the interval $[0,2 \pi)$,

$$
D(x)=\frac{\pi-x}{2 \pi}, \quad x \in[0,2 \pi) .
$$

It has the property that

$$
\int_{0}^{2 \pi} D(x) d x=0
$$

which we can write as $D \perp 1$. We then extend $D$ to a $2 \pi$ periodic function on the whole real line by letting

$$
D(x+2 k \pi)=D(x), \quad k \in \mathbb{Z} .
$$

This function is the Bernoulli monospline of degree 1. We refer the reader to [12] and [9] for more details about its properties.

Lemma 1. If $f \perp 1$ and $u=D * f$, then $u \perp 1$, and $u$ is the unique solution to the boundary value problem

$$
u^{\prime}(x)=f(x), \quad x \in(0,2 \pi), \quad u(0)=u(2 \pi) .
$$

Proof. Let $u=D * f$. We show first that $u \perp 1$. We have

$$
\int_{0}^{2 \pi} u(x) \mathrm{d} x=\int_{0}^{2 \pi}\left(\int_{0}^{2 \pi} D(x-y) d x\right) f(y) d y=0,
$$


since $D \perp 1$ and $D$ is $2 \pi$-periodic. Next we find the derivative of $u$. For $x \in[0,2 \pi)$, we split the integral defining $(D * f)(x)$ into two parts,

$$
u(x)=(D * f)(x)=\int_{0}^{2 \pi} D(x-y) f(y) d y=G(x)+H(x)
$$

where

$$
G(x)=\int_{0}^{x} D(x-y) f(y) d y=\int_{0}^{x} \frac{\pi-x+y}{2 \pi} f(y) d y
$$

and

$$
H(x)=\int_{x}^{2 \pi} D(x-y) f(y) d y=\int_{x}^{2 \pi} \frac{-\pi-x+y}{2 \pi} f(y) d y .
$$

Then we differentiate $G$ and $H$ with respect to $x$ and use the Leibniz integral formula,

$$
\begin{aligned}
\frac{d}{d x} G(x) & =\frac{1}{2} f(x)-\frac{1}{2 \pi} \int_{0}^{x} f(y) d y \\
\frac{d}{d x} H(x) & =\frac{1}{2} f(x)-\frac{1}{2 \pi} \int_{x}^{2 \pi} f(y) d y
\end{aligned}
$$

and it follows that $u^{\prime}(x)=f(x)$ due to the assumption that $f \perp 1$.

The boundary condition follows from

$$
u(2 \pi)-u(0)=\int_{0}^{2 \pi} u^{\prime}(x) \mathrm{d} x=\int_{0}^{2 \pi} f(x) \mathrm{d} x=0,
$$

since $f \perp 1$. To see that $u$ is unique, suppose $f=0$ in (5). Then $u$ must be a constant, but to satisfy $u \perp 1$ we must have $u=0$.

Remark: Observe that if $f$ is not orthogonal to 1 then $D * f=D *(I-Q) f$, where $Q$ denotes the $L^{2}$ projection onto the space of constant functions. We will make use of this in (8).

Using Lemma 1 we get the well-known representation of $A_{\text {per }}^{1}$ (see e.g. [12, p. 97]) as

$$
A_{\mathrm{per}}^{1}=\Pi_{0} \oplus \tilde{A}_{\mathrm{per}}^{1},
$$

with

$$
\tilde{A}_{\text {per }}^{1}=\{D * f:\|f\| \leq 1, \quad f \perp 1\} .
$$

Pinkus [12, p. 133] proved the following.

Theorem 1. The widths of $A_{\text {per }}^{1}$ are given by

$$
d_{2 m-1}\left(A_{p e r}^{1}\right)=d_{2 m}\left(A_{p e r}^{1}\right)=\frac{1}{m}, \quad m \geq 1 .
$$

Furthermore,

$$
[1, \sin (x), \cos (x), \ldots, \sin (m-1) x, \cos (m-1) x]
$$

is an optimal $(2 m-1)$-dimensional subspace for $d_{2 m-1}\left(A_{p e r}^{1}\right)$, and

$$
X_{2 m}=\left\{b+\sum_{j=0}^{2 m-1} b_{i} D(\cdot-j \pi / m): \quad \sum_{j=0}^{2 m-1} b_{j}=0\right\}
$$

is an optimal $2 m$-dimensional subspace for $d_{2 m}\left(A_{p e r}^{1}\right)$. 
From the definition of the Bernoulli monospline we find that

$$
D(x-j \pi / m)=-\frac{x}{2 \pi}+q_{j}(x), \quad j=0,1, \ldots, 2 m-1,
$$

where $q_{j}(x)$ is some piecewise constant function with a breakpoint at $j \pi / m$. The condition

$$
\sum_{j=0}^{2 m-1} b_{j}=0
$$

then ensures that the monomial $x$ is not in the space $X_{2 m}$. Thus, $X_{2 m}=S_{0, \boldsymbol{\tau} \text {,per }}$, with $\boldsymbol{\tau}$ as in (2).

Remark: As stated in the introduction, Pinkus proved a more general result than Theorem 1 . In fact, it

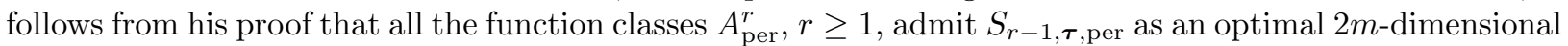
space for every $m \geq 1$, where $\boldsymbol{\tau}$ still is as in (2). Moreover, the $n$-widths of $A_{\text {per }}^{r}$ satisfy $d_{n}\left(A_{\text {per }}^{r}\right)=d_{n}\left(A_{\text {per }}^{1}\right)^{r}$.

Now, observe that if $Q$ is the $L^{2}$ projection onto the space of constant functions, then

$$
\{f:\|f\| \leq 1, f \perp 1\}=\{(I-Q) f:\|f\| \leq 1\}=(I-Q)(B),
$$

which follows from the fact that $\|(I-Q) f\| \leq\|f\|$. To prove optimality of higher order spline spaces, we let $K$ be the integral operator with the kernel

$$
K(x, y)=D(x-y), \quad x, y \in[0,2 \pi) .
$$

Then $K f=D * f$, and so using Lemma 1, $r$ times, together with (6), we have

$$
A_{\mathrm{per}}^{r}=\Pi_{0} \oplus \tilde{A}_{\mathrm{per}}^{r},
$$

where $\tilde{A}_{\text {per }}^{r}$ for $r \geq 1$, is defined by

$$
\tilde{A}_{\mathrm{per}}^{r}=K^{r}(B) .
$$

As was done in the proof of Lemma 1 one can use the definition of $D$ in (4) to obtain an explicit representation of the kernel of $K$,

$$
K(x, y)=\frac{1}{2 \pi} \begin{cases}-\pi-x+y, & x<y \\ \pi-x+y, & x \geq y\end{cases}
$$

If $K^{*}$ denotes the adjoint of $K$, then $K^{*}(x, y)=K(y, x)$, and so using (9) one can verify that $K^{*}=-K$. We are now ready to invoke Corollary 1.

Theorem 2. Suppose $r \geq 1$ and $\boldsymbol{\tau}$ is as in (2). Then $S_{d, \boldsymbol{\tau}, \text { per }}$ is an optimal $2 m$-dimensional spline space for the function class $A_{\text {per }}^{r}$ for all $d \geq r-1$ and $m \geq 1$.

Proof. Observe that $1 \in S_{d, \boldsymbol{\tau}, \text { per }}$, and so we can define the $(2 m-1)$-dimensional spaces $\tilde{S}_{d}$, by

$$
\tilde{S}_{d}=\left\{s \in S_{d, \boldsymbol{\tau}, \text { per }}: s \perp 1\right\} .
$$

Since the derivative of a spline is a spline on the same knot vector of one degree lower, it follows from

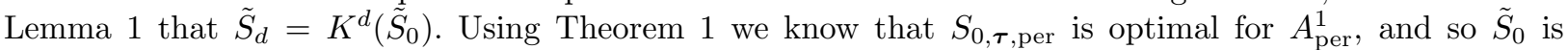
optimal for the set $\tilde{A}_{\text {per }}^{1}$.

It then follows from Corollary 1 , with $\tilde{S}_{0}$ playing the role of the $(2 m-1)$-dimensional space $X_{2 m-1}^{0}$, that $\tilde{S}_{d}$ is optimal for $\tilde{A}_{\text {per }}^{r}$ for all $d \geq r-1$. Lastly, since both $\tilde{S}_{d} \perp 1$ and $\tilde{A}_{\text {per }}^{r} \perp 1$, we conclude that $S_{d, \boldsymbol{\tau}, \mathrm{per}}=\Pi_{0} \oplus \tilde{S}_{d}$ is optimal for $A_{\mathrm{per}}^{r}=\Pi_{0} \oplus \tilde{A}_{\mathrm{per}}^{r}$ for all $d \geq r-1$. 


\subsection{Shifted knot vector}

Next, let us show that the optimality result generalizes to more general uniform knot vectors, formed by augmenting the knot vector $\tau$ in (2) with 0 and then shifting it to the right, provided we use the second kind of periodic spline space. To be precise, let $\boldsymbol{\xi}=\left(\xi_{1}, \ldots, \xi_{2 m}\right)$, where $\xi_{1} \in(0, \pi / m)$ and

$$
\xi_{j+1}=\xi_{j}+\frac{\pi}{m}, \quad j=1, \ldots, 2 m-1 .
$$

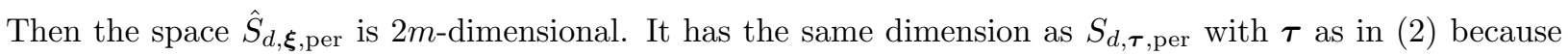
it has one more interior knot but also one more boundary condition.

Corollary 2. The $2 m$-dimensional space $\hat{S}_{d, \boldsymbol{\tau}, \text { per }}$ is optimal for $A_{p e r}^{r}$, for all $d \geq r-1$.

Proof. Any function $f \in A_{\text {per }}^{r}$ can be extended to a $2 \pi$ periodic function $\hat{f}$ on $\mathbb{R}$, similar to how we defined $D$ in (4). Since $f^{(r-1)}$ is continuous for $f \in A_{\text {per }}^{r}$, the restriction to $[0,2 \pi)$ of the shifted function $\hat{f}(\cdot-\delta)$ is also in $A_{\mathrm{per}}^{r}$ for all $\delta \in \mathbb{R}$ and $r \geq 1$. Then, since

$$
\|f-s\|=\|\hat{f}(\cdot-\delta)-\hat{s}(\cdot-\delta)\|,
$$

we can shift all the knots in the spline spaces in Theorem 2.

\section{Non-optimality of splines of odd dimension}

In this section we show that the only optimal spline space of odd dimension for $A_{\mathrm{per}}^{r}$ is the 1-dimensional space of constant functions. To prove this we make use of a theorem of Karlovitz [7] that provides a necessary condition for optimality.

Lemma 2. If $X_{2 m-1}$ is an optimal $(2 m-1)$-dimensional space for $A_{p e r}^{r}$, then every function in $X_{2 m-1}$ must be orthogonal to both $\sin (m x)$ and $\cos (m x)$.

Proof. First observe that if $X_{2 m-1}$ does not contain the constant functions then $E\left(A_{\mathrm{per}}^{r}, X_{2 m-1}\right)=\infty$ and so it cannot be optimal for $A_{\text {per }}^{r}$. Thus, if $X_{2 m-1}$ is an optimal $(2 m-1)$-dimensional space for $A_{\text {per }}^{r}$ we can write it as

$$
X_{2 m-1}=\Pi_{0} \oplus \tilde{X}_{2 m-2},
$$

and use (7) to conclude that $\tilde{X}_{2 m-2}$ is an optimal $(2 m-2)$-dimensional space for $\tilde{A}_{\mathrm{per}}^{r}$. Since $\tilde{A}_{\mathrm{per}}^{r}=K^{r}(B)$ it is well known $[12$, p. 6$]$ that the optimal space $\tilde{X}_{2 m-2}$ is extremal for the Rayleigh-Ritz characterization

$$
E\left(\tilde{A}_{\mathrm{per}}^{r}, \tilde{X}_{2 m-2}\right)=\inf _{Y_{2 m-2}} E\left(\tilde{A}_{\mathrm{per}}^{r}, Y_{2 m-2}\right)=\inf _{Y_{2 m-2}} \sup _{f \perp Y_{2 m-2}} \frac{\left(K^{r}\left(K^{r}\right)^{*} f, f\right)}{(f, f)}=\inf _{Y_{2 m-2}} \sup _{f \perp Y_{2 m-2}} \frac{\left(K^{2 r} f, f\right)}{(f, f)},
$$

and the result then follows from [7, Theorem 1] with $K^{2 r}$ playing the role of the operator $Q$ in [7].

Note that [7, Theorem 1] also implies that an optimal $2 m$-dimensional space for $A_{\text {per }}^{r}$ must only be orthogonal to a linear combination of $\sin (m x)$ and $\cos (m x)$, and not both at the same time. One can verify that this is true for the optimal spline spaces in Theorem 2 and Corollary 2.

Now let $\boldsymbol{\tau}=\left(\tau_{1}, \ldots, \tau_{m}\right)$ be the knot vector given as

$$
\tau_{j}=\frac{(2 j-1) \pi}{m}, \quad j=1,2, \ldots, m,
$$

and let $\hat{S}_{d, \boldsymbol{\tau}, \text { per }}$ be the $m$-dimensional spline space on this knot vector. 
Lemma 3. Suppose $\boldsymbol{\tau}$ is as given in (11). Then the space $\hat{S}_{d, \boldsymbol{\tau}, \text { per }}$ is the unique polynomial spline space of degree $d$ and dimension $\geq m$ (and among all possible boundary conditions) that is orthogonal to both $\sin (m x)$ and $\cos (m x)$.

Proof. We proceed by induction. First let $d=0$ and consider an arbitrary knot vector $\boldsymbol{\eta}=\left(\eta_{1}, \ldots, \eta_{k}\right)$ on the interval $[0,2 \pi]$. If the piecewise constant spline space $S_{0, \boldsymbol{\eta}}$ is orthogonal to both $\sin (m x)$ and $\cos (m x)$, then $\boldsymbol{\eta}$ must satisfy

$$
\int_{\eta_{j}}^{\eta_{j+1}} \sin (m x) \mathrm{d} x=\int_{\eta_{j}}^{\eta_{j+1}} \cos (m x) \mathrm{d} x=0,
$$

for all $j=1,2, \ldots, k$ (where $j=k$ corresponds to cyclic integration, i.e. first integrate from $\eta_{k}$ to $2 \pi$ then add the integral from 0 to $\eta_{1}$ ). Thus, $\boldsymbol{\eta}=\boldsymbol{\tau}$ in (11) and $S_{0, \boldsymbol{\eta}}=\hat{S}_{0, \boldsymbol{\tau} \text {,per }}$.

Now assume the result is true for degree $d$ and again consider an arbitrary knot vector $\boldsymbol{\eta}=\left(\eta_{1}, \ldots, \eta_{k}\right)$ on $[0,2 \pi]$. Let $S_{d+1, \boldsymbol{\eta}}$ be a spline space of degree $d+1$ on $\boldsymbol{\eta}$, with possibly reduced smoothness at the knots (repeated knots), that is orthogonal to both $\sin (m x)$ and $\cos (m x)$. For $s \in S_{d+1, \boldsymbol{\eta}}$ we have, using integration by parts on each knot interval, that

$$
\begin{aligned}
\int_{0}^{2 \pi} s(x) \cos (m x) \mathrm{d} x & =\frac{1}{m} \sum_{j=1}^{k}\left(s\left(\eta_{j}^{-}\right)-s\left(\eta_{j}^{+}\right)\right) \sin \left(m \eta_{j}\right)-\frac{1}{m} \int_{0}^{2 \pi} s^{\prime}(x) \sin (m x) \mathrm{d} x, \\
\int_{0}^{2 \pi} s(x) \sin (m x) \mathrm{d} x & =\frac{1}{m}\left[s(0)-s(2 \pi)+\sum_{j=1}^{k}\left(s\left(\eta_{j}^{+}\right)-s\left(\eta_{j}^{-}\right)\right) \cos \left(m \eta_{j}\right)\right] \\
& +\frac{1}{m} \int_{0}^{2 \pi} s^{\prime}(x) \cos (m x) \mathrm{d} x,
\end{aligned}
$$

with $s^{\prime} \in S_{d, \boldsymbol{\eta}}$. If we first consider the splines of maximal smoothness in $S_{d+1, \boldsymbol{\eta}}\left(s \in C^{d}\right)$ then, using the induction hypothesis, we find that $\boldsymbol{\eta}=\boldsymbol{\tau}$ in (11), since the derivative operator is onto in this case. If we now consider an arbitrary spline $s \in S_{d+1, \tau}$, we find that $s\left(\tau_{j}^{+}\right)=s\left(\tau_{j}^{-}\right)$and $s(0)=s(2 \pi)$. Thus, $S_{d+1, \eta}=\hat{S}_{d+1, \boldsymbol{\tau}, \mathrm{per}}$.

Theorem 3. No spline space of degree $d \geq 0$ is optimal for $d_{2 m-1}\left(A_{p e r}^{r}\right)$ when $m>1$.

Proof. Assume for the sake of contradiction that there is an optimal spline space for $d_{2 m-1}\left(A_{\text {per }}^{r}\right)$ of some degree $d$. From Lemma 2 we know that this space must be orthogonal to both $\sin (m x)$ and $\cos (m x)$, and so from Lemma 3 it has to be the $m$-dimensional space $\hat{S}_{d, \boldsymbol{\tau} \text {,per }}$, with $\boldsymbol{\tau}$ as in (11). However, this $m$-dimensional space cannot be optimal for $d_{2 m-1}\left(A_{\mathrm{per}}^{r}\right)$, since $d_{m}\left(A_{\mathrm{per}}^{r}\right)>d_{2 m-1}\left(A_{\mathrm{per}}^{r}\right)$ whenever $m>1$.

While the above theorem is a negative result, it is worth pointing out that the numerical experiments carried out in [3] indicate that periodic spline spaces of odd dimension are 'very close' to being optimal when their degree is high enough. This possibility is also supported by [4, Lemma 1], which essentially implies that the distance to $A_{\text {per }}^{r}$ from any periodic spline space can only improve, or at worst stay the same, as their degree increases.

\section{Conclusions}

The main conclusion to be drawn from this paper and the papers [4] and [5] is that imposing maximal smoothness on spline spaces does not impede approximation power as their degree increases. In fact, with the right knot vectors, these spaces can achieve optimal approximation. This holds true when approximating functions in Sobolev spaces with or without various natural boundary conditions. This agrees with various numerical results carried out in the IGA framework [6]. Specifically, in this paper we have shown that the space of periodic functions $H_{\mathrm{per}}^{r}(0,2 \pi)$ admits optimal even-dimensional spline spaces of all degrees $\geq r-1$. 
However, except for the trivial case that the dimension is one, optimality does not hold for odd-dimensional spline spaces. As mentioned in the introduction, optimality of the even-dimensional spline spaces has already been shown in many other periodic $L^{p}$-settings, but as far as we are aware it is an open question whether odd-dimensional spline spaces are optimal in any other case of $L^{p}$ approximation to periodic functions with bounded $r$ th derivative in $L^{q}$ for $p, q \neq 2$.

\section{Acknowledgements}

Espen Sande was supported by the European Research Council under the European Union's Seventh Framework Programme (FP7/2007-2013) / ERC grant agreement 339643.

\section{References}

[1] L. Beirão da Veiga, A. Buffa, G. Sangalli, and R. Vázquez, Mathematical analysis of variational isogeometric methods, Acta Numer. 23 (2014), 157-287.

[2] J. Austin Cottrell, Thomas J.R. Hughes, and Yuri Bazilevs, Isogeometric analysis: Toward integration of CAD and FEA, Wiley, 2009.

[3] J. A. Evans, Y. Bazilevs, I. Babuska, and T. J. R. Hughes, n-Widths, sup-infs, and optimality ratios for the k-version of the isogeometric finite element method, Comput. Methods Appl. Mech. Engrg. 198 (2009), $1726-1741$.

[4] M. S. Floater and E. Sande, Optimal spline spaces of higher degree for $L^{2} n$-widths, J. Approx. Theory 216 (2017), $1-15$.

[5] Optimal spline spaces for $L^{2} n$-width problems with boundary conditions, Constr. Approx. (2018).

[6] T. J. R. Hughes, J. A. Cottrell, and Y. Bazilevs, Isogeometric analysis: CAD, finite elements, NURBS, exact geometry and mesh refinement, Comput. Methods Appl. Mech. Eng. 194 (2005), 4135-4195.

[7] L. A. Karlovitz, Remarks on variational characterizations of eigenvalues and n-width problems, J. Math. Anal. Appl. 53 (1976), 99-110.

[8] A. Kolmogorov, Über die beste Annäherung von Funktionen einer gegebenen Funktionenklasse, Ann. of Math. 37 (1936), $107-110$.

[9] N. Korne ̌̌chuk, Exact constants in approximation theory, Cambridge University Press, Cambridge, 1991, Translated from Russian by K. Ivanov.

[10] A. A. Melkman and C. A. Micchelli, Spline spaces are optimal for $L^{2}$ n-width, Illinois J. Math. 22 (1978), $541-564$.

[11] A. Pinkus, On n-widths of periodic functions, J. Analyse Math. 35 (1979), 209-235.

[12] , n-Widths in approximation theory, Springer-Verlag, Berlin, 1985. 